\title{
Anatolii Borisovich Mikhailovskii (In Honor of His 75th Birthday)
}

DOI: $10.1134 / \mathrm{S} 1063780 \mathrm{X} 10120093$

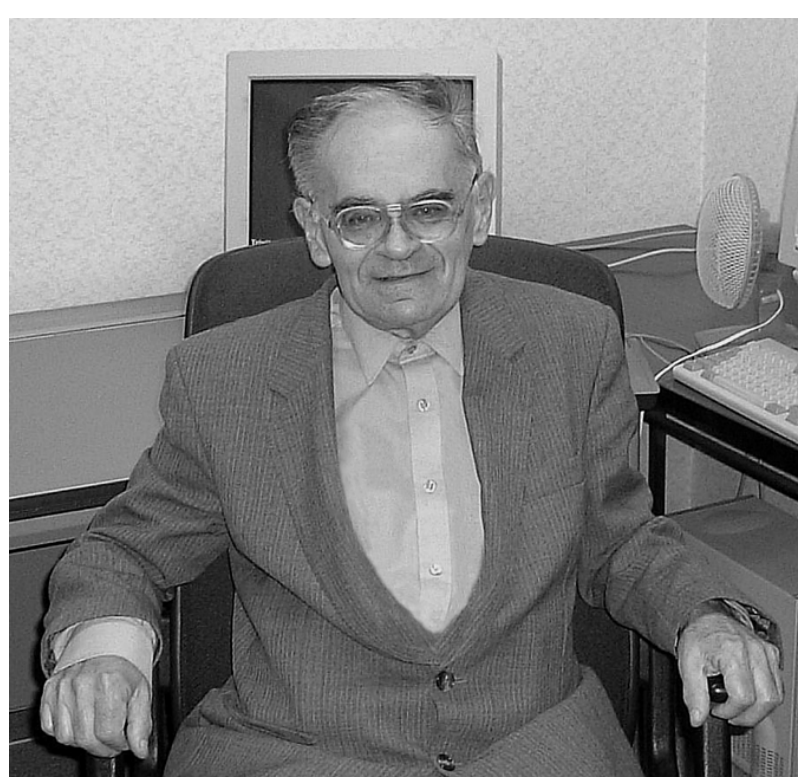

May 22, 2010, was the 75th birthday of Anatolii Borisovich Mikhailovskii, a corresponding member of the Russian Academy of Sciences, doctor of physical and mathematical sciences, professor, and principal researcher at the Department of Plasma Theory of the Institute of Tokamak Physics, Kurchatov Institute. Mikhailovskii is undoubtedly one of the world leaders in the field of theory of high-temperature magnetized plasma. He is one of the most acknowledged and most cited theorists in the plasma physics community. Mikhailovskii has written more than 360 papers published in the leading scientific journals, 5 monographs, and 13 reviews.

The main subject of Mikhailovskii's studies is numerous plasma instabilities, which are (themselves or in combination with anomalous processes induced by them) one of the most serious obstacles in achieving controlled thermonuclear fusion in reactors with magnetic plasma confinement. The books by Mikhailovskii became an encyclopedia of plasma instabilities for scientists specialized in the field of plasma physics and thermonuclear fusion.
Mikhailovskii predicted drift-cyclotron instability (together with A.V. Timofeev) and drift-Alfvén instability (together with L.I. Rudakov), which are two cornerstones of the modern theory of plasma instabilities. He developed a general approach to studying arbitrary types of instabilities of collisionless plasma in a magnetic field with straight field lines. He, together with his colleagues from M.S. Ioffe's laboratory, discovered the phenomenon of drift-conical instability, which leads to plasma ejection from magnetic mirror systems. Mikhailovskii, together with B.B. Kadomtsev and A.V. Timofeev, discovered negative energy waves in dispersive media. He formulated the concept of beam-drift instability and investigated other instabilities. He carried out a series of studies of collective processes in plasmas confined by a toroidal magnetic field, first of all, in tokamak plasmas. One of the classical results of these studies is the prediction (together with V.D. Shafranov) of the effect of self-stabilization of high-pressure plasma in toroidal systems.

Along with the theory of thermonuclear plasmas, Mikhailovskii contributed greatly to the theory of gravitating systems (together with A.M. Fridman), relativistic plasma astrophysics (together with R.Z. Sagdeev and J.G. Lominadze), and space physics (together with O.A. Pokhotelov).

In recent years, Mikhailovskii has worked on such issues as the development of numerical codes for modeling various instabilities in tokamak plasmas, theory of neoclassical magnetic islands resulting from tearing instability, theory of Alfvén modes excited by fast ions, and fundamental problems of the stability of rotating plasmas.

Mikhailovskii created a powerful scientific school. Among his pupils, there are 14 candidates of sciences, several doctors of sciences, and several academicians. Many of his pupils, some of whom are now working out of Russia, are also well recognized by the world thermonuclear community.

The Editorial Board of the journal Plasma Physics Reports, colleagues from the Department of Plasma Theory, and numerous friends and pupils of Anatolii Borisovich wish him health, happiness, and further fruitful scientific activity! 